\title{
A functional architecture of the human brain: Emerging insights from the science of emotion
}

\author{
Kristen A. Lindquist ${ }^{1,{ }^{*}}$ and Lisa Feldman Barrett ${ }^{2,3}$ \\ 1 University of North Carolina, Chapel Hill Department of Psychology Chapel Hill, 321 Davie Hall \\ North Carolina 27599 kristen.lindquist@unc.edu \\ 2 Northeastern University Department of Psychology 125 Nightingale Hall Northeastern University \\ Boston, MA 02115 I.barrett@neu.edu \\ ${ }^{3}$ Harvard Medical School/Massachusetts General Hospital/Martinos Center for Biomedical \\ Imaging Department of Psychiatry $14913^{\text {th }}$ St. Charlestown, MA 02129
}

\begin{abstract}
The 'faculty psychology' approach to the mind, which attempts to explain mental function in terms of categories that reflect modular 'faculties', such as emotions, cognitions, and perceptions, has dominated research into the mind and its physical correlates. In this paper, we argue that brain organization does not respect the commonsense categories belonging to the faculty psychology approach. We review recent research from the science of emotion demonstrating that the human brain contains broadly distributed functional networks that can each be re-described as basic psychological operations that interact to produce a range of mental states, including, but not limited to, anger, sadness, fear, disgust, and so on. When compared to the faculty psychology approach, this 'constructionist' approach provides an alternative functional architecture to guide the design and interpretation of experiments in cognitive neuroscience.
\end{abstract}

"A science of the relations of mind and brain must show how the elementary ingredients of the former correspond to the elementary functions of the latter," [1] (p. 28).

\section{Faculties v. Constructions: The Search for the Physical Basis of the Mind}

In the mid- $19^{\text {th }}$ century, philosophers, neurologists, and physiologists began the search for the physical basis of the human mind. Many subscribed to the view that the mind was divided into distinct mental categories reflecting modular "faculties," such as emotions (e.g., anger, disgust, fear, happiness, sadness, etc.), cognitions (e.g., memory, attention, decisions, etc.), and perceptions (e.g., visual images, auditory sounds, etc.). Each faculty, reflected in commonsense experiences (now called "folk psychology"), was assumed to have its own biological core responsible for its creation, such as a location in the brain or a pattern in the physiology of the body. At the dawn of psychological science, William James [1] and Wilhelm Wundt [2] warned that commonsense faculties should be abandoned as a scientific framework for the mind and instead argued for a "constructionist" model in which mental events are caused by a set of more basic, common operations or elements (e.g., hedonic

\footnotetext{
(C) 2012 Elsevier Ltd. All rights reserved.

*corresponding author .
}

Publisher's Disclaimer: This is a PDF file of an unedited manuscript that has been accepted for publication. As a service to our customers we are providing this early version of the manuscript. The manuscript will undergo copyediting, typesetting, and review of the resulting proof before it is published in its final citable form. Please note that during the production process errors may be discovered which could affect the content, and all legal disclaimers that apply to the journal pertain. 
feeling was thought to be a common element in emotions, but also in cognitions and perceptions) (see Box 1). Nonetheless, faculty psychology has remained the dominant approach for understanding the mind and its physical correlates. In this paper, we use the science of emotion and emerging trends in cognitive neuroscience to reveal how functional neuroimaging studies illuminate the pitfalls of a faculty psychology approach to understanding the human mind. We then outline how a modern constructionist model of the mind, in which mental states are created from more basic, domain-general processes, can better explain evidence from the neuroscience literature. We close by discussing how a constructionist model provides a new functional architecture of mind: brain correspondence to guide hypothesis testing in cognitive neuroscience.

\section{No Brain-Based Evidence for Emotion Faculties}

The science of emotion provides one of the clearest examples of faculty psychology over the past century. Even today, popular models assume that there are a set of emotion faculties that are biologically "basic," meaning that each type of emotion is supposed to have its own biological core [e.g., 3]. One compelling variant of this "basic emotion" hypothesis is that each emotion faculty has its own dedicated neural circuitry that is architecturally distinct, inborn, and shared with other animals [4]. Although early neuroimaging studies were interpreted as evidence for emotion faculties (e.g., amygdala and fear [5], insula and disgust [6], subgenual anterior cingulate cortex (sgACC) and sadness [7], the orbitofrontal cortex (OFC) and anger [7]), recent meta-analyses [8, 9] fail to demonstrate a consistent and specific relationship between activation in any one region of brain tissue and any one emotional faculty (e.g., the amygdala shows increased activity across experiences and perceptions of anger, disgust, fear, happiness and sadness [9]). Although the lack of specificity in meta-analytic findings may merely reflect the limitations of neuroimaging methods, these findings are convergent with behavior and peripheral nervous system activity in emotion, as well as single-cell recordings, electrical stimulation studies, and lesion studies: to date, no consistent and specific one-to-one correspondence have been observed between physical measurements and anger, sadness, fear, disgust, etc. (Box 2). Together, these findings point to a qualitatively different model of the mind.

\section{Emotions as Constructed Mental States}

Although a constructionist framework has been largely absent from cognitive neuroscience to date (but for recent examples $[10,11]$ ), it has made regular appearances in the psychological literature on emotion throughout the last century [12]. One key hypothesis that unites constructionist approaches to emotion is that instances of emotion emerge from basic, interacting psychological processes (operations, building blocks, or "ingredients"); each operation performs a domain-general psychological function that contributes not only to a variety of emotions, but also to the variety of cognitions and perceptions that people experience and the actions that they perform. Our own approach [9, 13, 14] (see Box 1) goes beyond the psychological level to make specific hypotheses about how a network-level description of brain organization can be used to investigate the mind's fundamental psychological building blocks [13]. These basic operations are akin to "mental state variables" [15] that characterize the brain states corresponding to mental events. Brain imaging studies make a unique contribution to discovering the basic operations of the mind because they non-invasively identify how networks within a living brain contribute to instances of fear, sadness, anger, or any mental state.

Based on behavioral and psychophysiological data, we proposed that emotional experiences and perceptions are constructed from a set of more basic psychological operations. These include (1) the feelings that derive from sensations within the body (or the represen tations 
of those interoceptive sensations), called core affect, which are associated with limbic and paralimbic brain regions [16] within a "salience network" (see Table 1); and, (2) sensations from the world (exteroceptive sensations) [13] that are associated with modal and heteromodal sensory cortices (see Table 1). We hypothesize that these sensations are continuously made meaningful as "situated conceptualizations" using associations from past experience, organized as emotion category knowledge [13] that we believe is associated with the midline cortical, lateral prefrontal and temporal regions within the "default network" [13, 14], as well as language-relevant brain regions (see Table 1). Furthermore, we hypothesized that executive control supported by a frontoparietal network is important for orchestrating and regulating this construction process (see Table 1). Our proposal is that making meaning of sensations is not specific to emotion - these are the processes that create all mental states. Two growing sources of neuroimaging evidence provide support for this constructionist approach.

\section{Meta-analyses suggest basic psychological operations in emotion}

A first source of neuroimaging evidence for our constructionist hypotheses comes from recent meta-analyses of task-related neuroimaging studies on emotion [8, 9, 17]. A close inspection of these meta-analyses not only reveals support for the specific predictions of our constructionist model, but also provides support for a constructionist approach more broadly, in that many of the brain regions consistently activated during emotional experiences and perceptions (see Figure 1) show consistent activation in meta-analyses of other mental phenomena (e.g., memory, theory of mind, inavigation, prospection [18], semantics [19], moral decision making, empathy, [20] and pain [21]). These findings are consistent with the hypothesis that brain regions are implementing basic psychological operations that are not specific to any emotion per se, or even to the category "emotion." For example, regions of the default network (see Table 1), which formed two functional clusters in our emotion meta-analysis [17], also show a consistent increase in activation in metaanalyses of empathy [20] semantic processing [19], moral judgments [20], mentalizing about other people [18, 20], autobiographical memory [18], and imagining the future [18]. Limbic and paralimbic regions within "limbic" and "salience" networks (involved in visceromotor control and affective representation; see Table 1), formed two functional clusters in our meta-analysis [17] and also show consistent increases in activation within meta-analyses of pain [22], aversion [23], and across studies of attention, and language [24]. Aspects of a frontoparietal network formed a functional cluster in our meta-analysis of emotion [17] and shows consistent increases in meta-analyses of response inhibition, working memory and cognitive control [25] but also morality, empathy, theory of mind [20] and semantics [19] (see Table 1). Finally, a functional cluster of visual regions (e.g., lateral occipital cortex, MT ,+ V4) was observed in our meta-analysis [17], indicating that exteroceptive perception is an important operation in emotion.

\section{Intrinsic networks suggest basic psychological operations in emotion}

Recent evidence from task-independent studies of intrinsic connectivity are a second source of evidence in support of constructionist framework. Intrinsic functional networks spanning subcortical regions and frontal, cingulate, parietal, temporal, and occipital cortices [26, 27] are observed when researchers correlate low-frequency blood-oxygenation level dependent (BOLD) signal fluctuations in voxels when the brain is not being probed by an external stimulus (i.e., during task-independent periods). Such intrinsic (i.e., non-stimulus driven) activity accounts for a large proportion of the brain's metabolic budget [28], corresponds to anatomical connectivity between brain regions $[29,30]$, and is similar to the activity observed during psychological tasks [26,31], leading to the hypothesis that the structure of this activity reveals something about the basic functional architecture of the brain. Furthermore, individual differences in intrinsic connectivity predict activity during 
psychological tasks [32] and differences in behavior [31, 33, 34]. Perhaps most importantly, the intrinsic networks identified in the literature resemble the task-related activations observed in our emotion meta-analyses [9, 17], as well as task-related activations that occur in other psychological domains (e.g., memory [18], semantics [19], theory of mind [18, 20], pain [21], working memory [25]). See Table 1 for a list of networks derived in multiple intrinsic connectivity studies using multiple methods and their hypothesized functional description according to our constructionist framework. We focus on the seven intrinsic networks identified by Yeo et al. [27], since these networks are consistent with findings from other studies (see Table 1) and were derived from one of the largest sample sizes to date $(\mathrm{N}=1000)$.

Although methodology can impact the exact spatial topography and number of networks observed in a given study, there is still surprising consistency in the networks observed across individuals, within an individual across instances, and across methods [35]. Even studies that differ in the number of networks identified are still often consistent with one another, insofar that they merely represent more refined or expanded versions of the same canonical networks. For instance, analyses that observe more networks [e.g., a 7 v. 17 network solution, 27] or that use methods to optimize the temporal (as opposed to spatial) independence of networks [36] find that the canonical "default network" breaks down into two sub-networks: a cortical midline network implicated in autobiographical memory and simulation plus a lateral frontal and temporal network implicated in language $[27,36]$. This finding is still ultimately consistent with our constructionist hypothesis that the default network supports conceptualization, where conceptual knowledge is supported (at least in part) by language.

\section{A Constructionist Functional Architecture of the Human Brain}

Although there are many different levels of brain organization that can be meaningfully interpreted, we propose that psychological descriptions map best to the network level-the broadly distributed brain networks that are consistently evident in meta-analyses of taskrelated data and that consistently appear as intrinsic networks in task-independent data. We have proposed one specific set of constructionist hypotheses from our work on emotion, but a variety of hypotheses are possible (and hopefully, in the future, likely). As an alternative functional architecture of the human brain, a constructionist framework proposes that instances of mental categories from faculty psychology (which currently defines Western philosophy of mind) do not reflect the causal structure of the mind. Instead, instances of emotion, cognition, perception, and action are products of the mind that are realized from a set of more basic psychological operations that can plausibly describe the functions of broadly distributed networks in the human brain. It is tempting to assume that these networks are rigid, "Lego-like" building blocks, but we instead work with the idea that networks are flexible assemblies of neurons that statistically fire together in a probabilistic way. At present, data does not exist to allow a more specific mechanistic understanding of how proposed basic psychological operations emerge from the interplay of these neurons (e.g., at the level of interaction between neurons within different layers of cortex), although we hope this is a direction of future research. Understanding how psychological function emerges from assemblies of neurons is not a problem specific to constructionism, but exists for any neural model that hypothesizes emergentism.

Our constructionist proposal is synergistic with other contemporary themes in the cognitive neuroscience literature, particularly discussions on the limitations of faculty psychology and modular views of brain organization [37-40], network-based models of cognition [41, 42], and systems neuroscience more generally. Our proposal builds on these themes by suggesting that brain networks perform basic psychological operations that contribute to the 
construction of a number of different categories of mental phenomena that people experience differently (just as flour, water, and salt contribute to a variety of different recipes whose products look, taste, and feel differently). Finally, like others, we recognize the promise of understanding the functions of intrinsic connectivity networks [30, 35]. Yet we do not hypothesize that intrinsic networks correspond to faculties (i.e., particular emotions, cognitions or aspects of perception) [31], particular behaviors [30], or certain classes of stimuli [43].

The need for a unifying framework to understand how psychological functions map to physical measurements is not new-every scientist who uses psychological terms to describe physical measurements is using a psychological theory, whether explicitly or not $[44,45]$. Of late, there have been calls in cognitive neuroscience for a new mental ontology to guide the study and interpretation of brain function in psychological terms [25, 46, 47]. Construction provides one fruitful alternative. The value of a constructionist ontology can already be observed in research on memory, where there is an emerging recognition that interacting operations contribute to the construction of memories, and that some of these operations are important to other kinds of mental states (e.g., "cognitive" states such as fictitious imaginings, navigation, prospection, theory of mind, etc.)[11]. Yet, there is now evidence that these operations are also consistently engaged in emotion [9] and in perception [48]. A constructionist functional architecture explicitly builds on such observations and uses them to understand brain function. It thus encourages a qualitative shift in theorizing, from hypothesizing "where" different types of mental states "live" in the brain to asking "how" [49] networks of brain regions contribute to the construction of an instance of fear, sadness, anger, autobiographical memory, concentration, self-esteem, etc., as the variety of mental states that populate human mental life.

As an approach to cognitive neuroscience, a constructionist framework motivates important shifts in how research findings are interpreted, potentially helping to resolve the current limitations in reverse inference [50]. Researchers would no longer be free to interpret the functions of a brain region or network in terms of their own goals and interests, and would be compelled to consider other domains of research (including anatomy) during the inference process. For example, the anterior insula has been interpreted as a brain region dedicated to emotion, pain, attention, and memory. From a faculty psychology standpoint, the anterior insula might be interpreted as a multifunctional area, with the specificity of findings limited by current neuroimaging methods (which could presumably not detect the smaller anatomical circuits for each faculty within this broader brain region). From a constructionist approach, however, using intrinsic connectivity and anatomical findings as a guide [34, 51], the anterior insula is interpreted as a region that is necessary for two basic operations: (1) representing interoceptive sensations to regulate attention (in its dorsal extent); and, (2) creating affective feeling (in its ventral extent) which, according to Wundt [2], is a core feature of all mental states. In a construction framework, the goal is to describe the psychological "lowest common denominators" or psychological primitives [13] that will link a range of findings across mental states that appear to be very different on the surface. The anterior insula might thus be a "hot spot" in the human brain that shows increased activity during a range of perceptual, cognitive, and emotional events [24] because interoceptive information from the body is an important ingredient in all these mental states. Using these psychological primitives, a constructionist approach might be profitably incorporated into the Cognitive Atlas project, which is a tool for mapping brain activity to psychological constructs [25, 52].

A constructionist approach would not only influence the interpretation of findings, but it also has value for shaping how neuroimaging experiments are conducted in the first place. Rather than starting with a commonsense category and looking for its neural correlates, 
experiments would manipulate specific psychological operations in an a priori way, and then examine their consequences for the content and type of resulting mental states [for a recent example of this approach, see 53].

Of course, the search for psychological primitives is just beginning. One thing to consider moving forward is whether the basic elements we have hypothesized in this paper and elsewhere $[9,13]$ do in fact represent the most "psychologically primitive" level of description (i.e., or whether they, themselves, can be reduced to something more psychologically basic). For example, the limbic circuitry that is important for creating core affect can be parsed further into networks supporting affiliation or avoidance [54] and these networks themselves contain more circumscribed circuits that are linked to specific actions (e.g., freezing, fleeing, and fighting) [55]. Yet such actions are not synonymous with the complex human psychological categories (e.g., fear and anger) that we are trying to understand [14, 55], and which might be better approached using a construction framework. Neuroimaging methods do not have the spatial or temporal resolution to permit strong conclusions about the specificity of all neural mechanisms, although we suggest that these methods are valuable for mapping the distributed, large-scale brain networks whose functions are best described in psychological terms.

A successful science of the mind depends not only on having sophisticated measurements of the brain, but also on a theoretical framework to link those data to psychological constructs in a meaningful and generative way. A constructionist approach requires that cognitive neuroscientists abandon the naïve realist assumption that the structure of experience (emotions, cognitions, and perceptions) reveals causal mechanisms within the brain. Constructionism distinguishes psychological operations from the mental events that they produce and provides the field with an alternative for mapping mind to brain.

\section{Acknowledgments}

We thank Christy Wilson-Mendenhall for her comments on a previous draft of this manuscript and are especially grateful for her thoughts on the default network's role in conceptual processing. Preparation of this manuscript was supported by a Harvard University Mind/Brain/Behavior Postdoctoral Fellowship to Kristen Lindquist and a National Institute of Health Director's Pioneer Award (DP1OD003312) to Lisa Feldman Barrett.

\section{Glossary}

Faculty psychology

Natural kind

Constructionism
A scientific framework in which it is hypothesized that commonsense mental states such as cognitions (e.g., memory, attention, decisions, etc.), emotions (e.g., anger, disgust, fear, happiness, sadness, etc.), and perceptions (e.g., visual images, auditory sounds, etc.), are the basic faculties or abilities of the mind. A faculty psychology approach assumes that these categories can be mapped to the physical structure of the body and brain.

A phenomenon that exists in nature independently of human perception. In the philosophical literature, a phenomenon can be defined as a natural kind by homology (in that all instances of that phenomenon derive from the same causal mechanism) or analogy (in that all instances of that phenomenon share similar surface features; i.e., look the same, function the same).

A scientific framework in which it is hypothesized that commonsense mental states such as cognitions (e.g., memory, 


\section{Situated conceptualization}

\section{Core affect}

\section{Exteroceptive sensation}

Conceptualization

\section{Executive control}

attention, decisions, etc.), emotions (e.g., anger, disgust, fear, happiness, sadness, etc.), and perceptions (e.g., visual images, auditory sounds, etc.) are mental states that are created (or "constructed") out of the combination of more basic psychological operations or "ingredients." A constructionist approach assumes that these operations can be mapped to intrinsic networks in the brain.

The process of making sensations meaningful in a context using past experience.

The representation of sensations from inside the body that can be experienced as a bodily symptom or as feelings of pleasuredispleasure with some degree of arousal.

The representation of sensations from outside the body (e.g., visual, auditory, tactile, and olfactory sensations).

Representations of prior experiences (i.e., memories, knowledge) that are used to make meaning of sensations in the present.

A source of attention that helps prioritize sensations and conceptualizations for conscious awareness by selecting some information and inhibiting other information.

\section{References}

1. James, W. The principles of psychology. Holt; 1890.

2. Wundt, W. Outlines of psychology. Thoemmes Press; 1897/1998.

3. Ekman P, Cordaro D. What is meant by calling emotions basic. Emotion Review. 2011; 3:364-370.

4. Panksepp, J. Affective neuroscience: The foundations of human and animal emotions. Oxford University Press; USA: 2004.

5. Sprengelmeyer R, et al. Knowing no fear. Proc. Roy. Soc. B: Biolog. Sci. 1999; 266:2451-2451.

6 . Wicker B, et al. Both of us disgusted in my insula The common neural basis of seeing and feeling disgust. Neuron. 2003; 40:655-664. [PubMed: 14642287]

7. Murphy FC, et al. Functional neuroanatomy of emotions: A meta-analysis. Cog. Aff. \& Behav. Neurosci. 2003; 3:207-233.

8. Vytal K, Hamann S. Neuroimaging support for discrete neural correlates of basic emotions: a voxelbased meta-analysis. J. Cogn. Neurosci. 2010; 22:2864-2885. [PubMed: 19929758]

9. Lindquist KA, et al. The brain basis of emotion: A meta-analytic review. Behav. Brain Sci. 2012; 35:121-143. [PubMed: 22617651]

10. Roy M, et al. Ventromedial prefrontal-subcortical systems and the generation of affective meaning. Trends Cog. Sci. 2012; 16:147-156.

11. Schacter DL, et al. Remembering the past to imagine the future: the prospective brain. Nat. Rev. Neurosci. 2007; 8:657-661. [PubMed: 17700624]

12. Gendron M, Barrett LF. Reconstructing the past: A century of ideas about emotion in psychology. Emo. Rev. 2009; 1:316-316.

13. Barrett LF. The Future of Psychology: Connecting Mind to Brain. Persp. Psychol. Sci. 2009; 4:326-339.

14. Barrett LF. Emotions are real. Emotion. 2012; 12:413-429. [PubMed: 22642358]

15. Salzman CD, Fusi S. Emotion, cognition, and mental state representation in amygdala and prefrontal cortex. Annu. Rev. Neurosci. 2010; 33:173-202. [PubMed: 20331363]

16. Barrett LF, Bliss-Moreau E. Affect as a psychological primitive. Adv. Exp. Soc. Psychol. 2009; 41:167-218. [PubMed: 20552040] 
17. Kober H, et al. Functional grouping and cortical-subcortical interactions in emotion: a metaanalysis of neuroimaging studies. Neuroimage. 2008; 42:998-1031. [PubMed: 18579414]

18. Spreng RN, et al. The common neural basis of autobiographical memory, prospection, navigation, theory of mind, and the default mode: A quantitative metaanalysis. J. Cogn. Neurosci. 2009; 21:489-510. [PubMed: 18510452]

19. Binder JR, et al. Where is the semantic system? A critical review and meta-analysis of 120 functional neuroimaging studies. Cereb. Cortex. 2009; 19:2767-2796. [PubMed: 19329570]

20. Bzdok D, et al. Parsing the neural correlates of moral cognition: ALE meta-analysis on morality, theory of mind and empathy. Brain Struc. Func. (in press). DOI: 10.1007/s00429-012-0380-y.

21. Yarkoni T, et al. Large-scale automated synthesis of human functional neuroimaging neuroimaging data. Nat. Methods. 2011; 8:665-670. [PubMed: 21706013]

22. Lamm C, et al. Meta-analytic evidence for common and distinct neural networks associated with directly experienced pain and empathy for pain. Neuroimage. 2010; 54:2492-2502. [PubMed: 20946964]

23. Hayes D, Northoff G. Identifying a network of brain regions involved in aversion-related processing: A cross-species translational investigation. Front. Integrat. Neurosci. 2011; 5:1-21.

24. Nelson SM, et al. Role of the anterior insula in task-level control and focal attention. Brain Struct. Funct. 2010; 214:669-680. [PubMed: 20512372]

25. Lenartowicz A, et al. Towards an ontology of cognitive control. Top. Cogn. Sci. 2010; 2:678-692.

26. Smith SM, et al. Correspondence of the brain's functional architecture during activation and rest. Proc. Nat. Acad. Sci. 2009; 106:13040-13045. [PubMed: 19620724]

27. Yeo BT, et al. The Organization of the Human Cerebral Cortex Estimated By Functional Connectivity. J. Neurophysiol. 2011; 106:1125-1165. [PubMed: 21653723]

28. Raichle ME, Minton MA. Brain work and brain imaging. Annu. Rev. Neurosci. 2006; 29:449-476. [PubMed: 16776593]

29. Fox MD, Raichle ME. Spontaneous fluctuations in brain activity observed with functional magnetic resonance imaging. BOLD. 2007; 1:0-5.

30. Deco G, et al. Emerging concepts for the dynamical organization of resting-state activity in the brain. Nat. Rev. Neurosci. 2010; 12:43-56. [PubMed: 21170073]

31. Laird AR, et al. Behavioral interpretations of intrinsic connectivity networks. J. Cogn. Neurosci. 2011; 23:4022-4037. [PubMed: 21671731]

32. Mennes $\mathrm{M}$, et al. Inter-Individual differences in resting state functional connectivity predict taskinduced BOLD activity. Neuroimage. 2010; 50:1690-1701. [PubMed: 20079856]

33. Seeley WW, et al. Dissociable intrinsic connectivity networks for salience processing and executive control. J. Neurosci. 2007; 27:2349-2349. [PubMed: 17329432]

34. Touroutoglou A, et al. Dissociable large-scale networks anchored in the anterior insula subserve affective experience and attention/executive function. Neuroimage. 2012; 60:1947-1958. [PubMed: 22361166]

35. Van Dijk KR, et al. Intrinsic functional connectivity as a tool for human connectomics: theory, properties, and optimization. J. Neurophysiol. 2010; 103:297-321. [PubMed: 19889849]

36. Smith SM, et al. Temporally-independent functional modes of spontaneous brain activity. Proc. Nat. Acad. Sci. 2012; 109:3131-3136. [PubMed: 22323591]

37. Pessoa L. On the relationship between emotion and cognition. Nat. Rev. Neurosci. 2008; 9:148158. [PubMed: 18209732]

38. Shackman AJ, et al. The integration of negative affect, pain, and cognitive control in the cingulate cortex. Nat. Rev. Neurosci. 2011; 12:154-167. [PubMed: 21331082]

39. Lewis MD. Bridging emotion theory and neurobiology through dynamic systems modeling. Behav. Brain Sci. 2005; 28:169-245. [PubMed: 16201458]

40. Uttal, WR. The new phrenology: The limits of localizing cognitive processes in the brain. The MIT Press; 2001.

41. Mesulam MM. From sensation to cognition. Brain. 1998; 121:1013-1013. [PubMed: 9648540]

42. Goldman-Rakic PS. Topography of cognition: Parallel distributed networks in primate association cortex. Annu. Rev. Neurosci. 1988; 11:137-156. [PubMed: 3284439] 
43. Northoff G. Immaual Kant's mind and the brain's resting state. Trends Cog. Sci. 2012; 16:356359.

44. Barrett LF. Bridging token identity theory and supervenience theory through psychological construction. Psychol. Inq. 2011; 22:115-127. [PubMed: 21785534]

45. Cacioppo J, Tassinary LG. Inferring psychological significance from physiological signals. Am. Psychol. 1990; 45:16-28. [PubMed: 2297166]

46. Gonsalves BD, Cohen NJ. Brain imaging, cognitive processes, and brain networks. Persp. Psychol. Sci. 2010; 5:744-752.

47. Decety J, Cacioppo J. Frontiers in human neuroscience: The golden triangle and beyond. Persp. Psychol. Sci. 2010; 5:767-771.

48. Bar M. The proactive brain: memory for predictions. Phil. Trans. Roy. Soc. B, Biolog. Sci. 2009; 364:1235-1243.

49. Shimamura AP. Bridging psychological and biological science: The good, bad, and ugly. Persp. Psychol. Sci. 2010; 5:772-775.

50. Poldrack RA. Can cognitive processes be inferred from neuroimaging data? Trends Cog. Sci. 2006; 10:59-63.

51. Craig ADB. How do you feel--now? The anterior insula and human awareness. Nat. Rev. Neurosci. 2009; 10:59-70. [PubMed: 19096369]

52. Poldrack RA. Mapping mental function to brain structure: How can cognitive neuroimaging succeed? Persp. Psychol. Sci. 2010; 5:753-761.

53. Oosterwijk S, et al. States of mind: Emotions, body feelings, and thoughts share distributed neural networks. Neuroimage. 2012; 62:2110-2128. [PubMed: 22677148]

54. Bickart $\mathrm{KC}$, et al. Intrinsic amygdala-cortical functional connectivity predicts social network size in humans. J. Neurosci. (in press). DOI: 10.1523/JNEUROSCI.1599-12.2012.

55. LeDoux J. Rethinking the emotional brain. Neuron. 2012; 73:653-676. [PubMed: 22365542]

56. Chomsky N. Rules and Representations. Behav. Brain Sci. 1980; 3:1-61.

57. Gall, FJ.; Spurzheim, JG. Outlines of the physiological system of Drs. Gall and Spurzheim: Indicating the dispositions and manifestations of the mind. Baldwin. Cradock \& Joy; 1815.

58. Barsalou LW. Simulation, situated conceptualization, and prediction. Phil. Trans. Roy. Soc. B: Biolog. Sci. 2009; 364:1281-1289.

59. Ekman P, Friesen WV. Constants across cultures in the face and emotion. J. Pers. Soc. Psychol. 1971; 17:124-129. [PubMed: 5542557]

60. Scherer KR, et al. Vocal cues in emotion encoding and decoding. Mot. and Emo. 1991; 15:123148.

61. Levenson RW. Blood, sweat, and fears: The autonomic architecture of emotion. Ann. N. Y. Acad. Sci. 2003; 1000:348-366. [PubMed: 14766648]

62. Barrett LF, et al. Of mice and men: Natural kinds of emotions in the mammalian brain? A response to Panksepp and Izard. Persp. Psychol. Sci. 2007; 2:297-297.

63. Mauss IB, Robinson MD. Measures of emotion: A review. Cogn. \& Emo. 2009; 23:209-237.

64. Grillner S, et al. Mechanisms for selection of basic motor programs--roles for the striatum and pallidum. Trends Neurosci. 2005; 28:364-370. [PubMed: 15935487]

65 . Vincent JL, et al. Evidence for a frontoparietal control system revealed by intrinsic functional connectivity. J. Neurophysiol. 2008; 100

66. Bartels A, Zeki S. The neural basis of romantic love. Neuroreport. 2000; 11:3829-3834. [PubMed: 11117499]

67. Bianchi-Demicheli F, Ortigue S. Toward an understanding of the cerebral substrates of woman's orgasm. Neuropsychologia. 2007; 45:2645-2659. [PubMed: 17543356]

68. Northoff G, Bermpohl F. Cortical midline structures and the self. Trends Cog. Sci. 2004; 8:102107.

69. Bar M. Visual objects in context. Nat. Rev. Neurosci. 2004; 5:617-629. [PubMed: 15263892]

70. Andrews-Hanna JR, et al. Evidence for the default network's role in spontaneous cognition. J. Neurophysiol. 2010; 104:322-322. [PubMed: 20463201] 
71. Wager TD, et al. Prefrontal-subcortical pathways mediating successful emotion regulation. Neuron. 2008; 59:1037-1050. [PubMed: 18817740]

72. Wager TD, et al. Placebo-induced changes in FMRI in the anticipation and and experience of pain. Science. 2004; 303:1162-1162. [PubMed: 14976306]

73. Crone EA, et al. Neural evidence for dissociable components of taskswitching. Cereb. Cortex. 2006; 16:475-486. [PubMed: 16000652]

74. Fan J, et al. The activation of attentional networks. Neuroimage. 2005; 26:471-479. [PubMed: 15907304]

75. Sakai K, Passingham RE. Prefrontal set activity predicts rule-specific neural processing during subsequent cognitive performance. J. Neurosci. 2006; 26:1211-1218. [PubMed: 16436608]

76. Corbetta M, et al. Neural systems for visual orienting and their relationships to spatial working memory. J. Cogn. Neurosci. 2002; 14:508-523. [PubMed: 11970810]

77. Morosan P, et al. Human primary auditory cortex: Cytoarchitectonic subdivisions and mapping into a spatial reference system. Neuroimage. 2001; 13:684-701. [PubMed: 11305897]

78. Eickhoff SB, et al. Segregation of visceral and somatosensory afferents: An fMRI and cytoarchitectonic mapping study. Neuroimage. 2006:1004-1014. [PubMed: 16529950]

79. Engel SA, et al. fMRI of human visual cortex. Nature. 1994; 369

80. Wager, TD., et al. The neuroimaging of emotion. In: Lewis, M., et al., editors. Handbook of Emotions. 2nd edn. Guilford; 2008. p. 513-530.

81. Wilson-Mendenhall CD, et al. Grounding emotion in situated conceptualization. Neuropsychologia. 2011; 49:1105-1127. [PubMed: 21192959] 


\section{Box 1}

\section{Faculty Psychology and Constructionism: Competing approaches to the mind}

In "faculty psychology," the mind is thought to consist of functionally encapsulated mental faculties or "mental organs" (akin to the organs of the body [56]), each with a specific and distinct physical cause [57]. In modern neuroscience, this view manifests as the hypothesis that a specific faculty corresponds consistently and specifically to increased activity in a given brain area, network of areas, or even in specific neurons.

Both James [1] and Wundt [2] were highly critical of faculty psychology as a scientific framework, arguing that faculties encoded in language and reflecting commonsense experiences (e.g., "anger," "disgust," "fear," "thoughts," "memory") were unlikely to represent the organization of the mind and could not support scientific induction. They instead hypothesized that mental states were constructed of more basic, domain-general "ingredients" of the mind that contributed to all mental states (including emotions, cognitions and perceptions). Despite the predominance of faculty psychology in modern psychology and neuroscience, important traces of this constructionist framework survived, especially in the science of emotion [12]. Our modern constructionist model [9, $13,14]$ was originally developed from experiential, behavioral, and peripheral physiological data. Rather than assuming that anger, disgust, fear, etc. are natural kind categories that are inborn and given by the structure of the brain and body, we propose that emotional experiences and perceptions are constructed from a set of more basic operations: sensations and feelings from the body (called core affect) and sensations from the world (exteroceptive sensations) that are made meaningful using past experience (conceptual knowledge), including knowledge about the emotion categories encoded in language $[9,13]$. The process of using knowledge to make meaning of sensations in context is called a "situated conceptualization" [58]. We hypothesize that executive control is important as an additional operation involved in regulating the process of situated conceptualization, insofar that it inhibits or prioritizes the representation of certain conceptual or sensory information in a given instance to create a unified conscious field. Constructionist views are also consistent with systems neuroscience approaches that conceive of mental states as emerging from the combination of brain networks (see Figure below). 


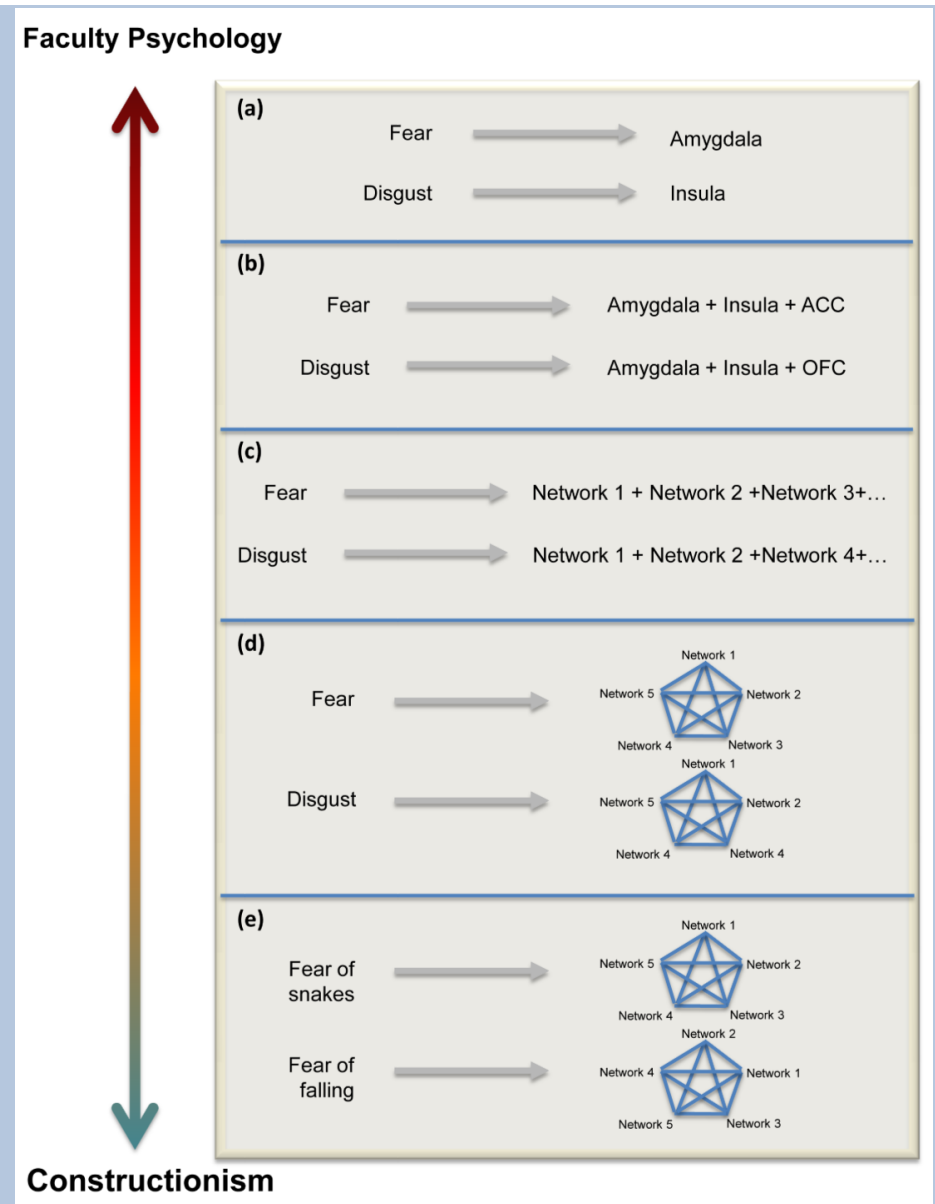

Box 1 Figure. Faculty psychology v. constructionist predictions for mapping mind to brain 


\section{Box 2}

\section{Evidence for Emotion Faculties in the Body?}

In the century preceding the development of neuroimaging methods, psychologists sought consistent and specific physical evidence for anger, disgust, fear, and other emotion faculties in physical measurements of the body such as human facial muscle movements (i.e., the hypothesis that certain emotions have consistent and specific facial expressions [59]), vocal acoustics (i.e., the hypothesis that certain emotions have consistent and specific physical properties of the voice [60]), or in coordinated patterns of autonomic response (i.e., the hypothesis that certain emotions have autonomic "signatures" [61]). Yet, starting in the early 1900's, review papers began observing that experimental evidence routinely fails to show that emotions can be distinguished by consistent and specific patterns in physical measurements of the human body [12]. In addition, we have recently pointed out that the lesion [9], single-cell recording [9] and human electrical brain stimulation [62] evidence in non-human and human animals is not consistent with emotional faculties either. While the careful use of lesions, optigenetics, etc., has identified circuits for individual actions (such as freezing, fleeing, and fighting), these actions are not synonymous with human emotion and don't constitute evidence for its biological basis [14, 55]. While some researchers continue to believe that better methods and more precise measures will reveal the physical basis of anger, sadness, fear, etc. many psychologists have come to the conclusion that physical measurements of the face, voice, and body index general affective dimensions (e.g., whether a person is in a pleasant or unpleasant state [63] or is aroused or quiescent), but not whether a person is angry, sad or afraid. Individual studies that support the idea of emotion faculties exist in a sea of experiments that do not replicate these findings, so that collectively, the empirical evidence shows that the mental states people call anger (or sadness, fear, or other emotions) in humans are too variable to hold natural kind status [13, 63]. These findings have exposed an explanatory gap in the science of emotion, because it is still necessary to explain how anger, or sadness, or fear emerges from the more general affective dimensions indexed by face, voice, and body measurements, and ultimately, from the brain. A constructionist approach is useful in this regard because it describes how these experiences emerge without assuming that the experiences themselves reflect faculties that correspond to the organizational structure of the brain or body. 


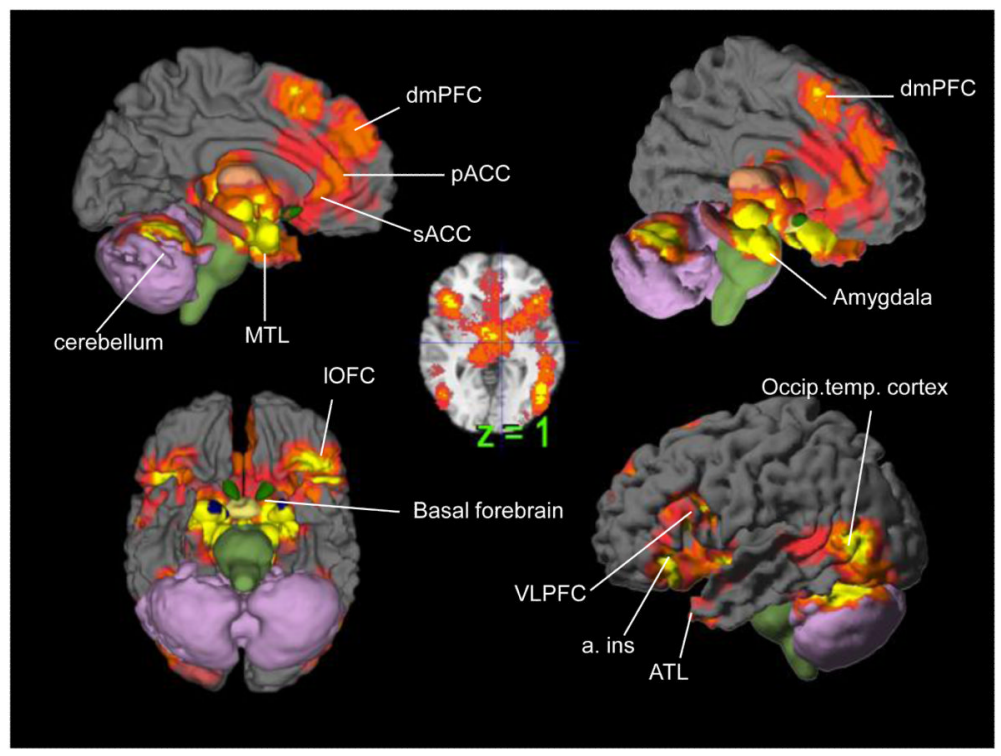

Figure 1. Meta-analytic findings of emotion

Brain areas with a consistent increase in activity across studies of emotion are shown in yellow, orange, pink and magenta (corresponding to height and three extent-based thresholds, respectively). Data are from the largest meta-analysis of studies of emotion experience and perception to date (91 studies published between January, 1990- December, 2007) and demonstrate that emotions are implemented by a range of brain regions including those associated with the default network (medial prefrontal cortex, medial temporal cortex, ventrolateral prefrontal cortex), salience network (insular cortex, anterior cingulate cortex, amygdala) and frontoparietal network (ventrolateral prefrontal cortex). (C) Cambridge University Press 2012. Reprinted with permission from [9]. 
Table 1

Intrinsic Networks and their functional description in our constructionist framework

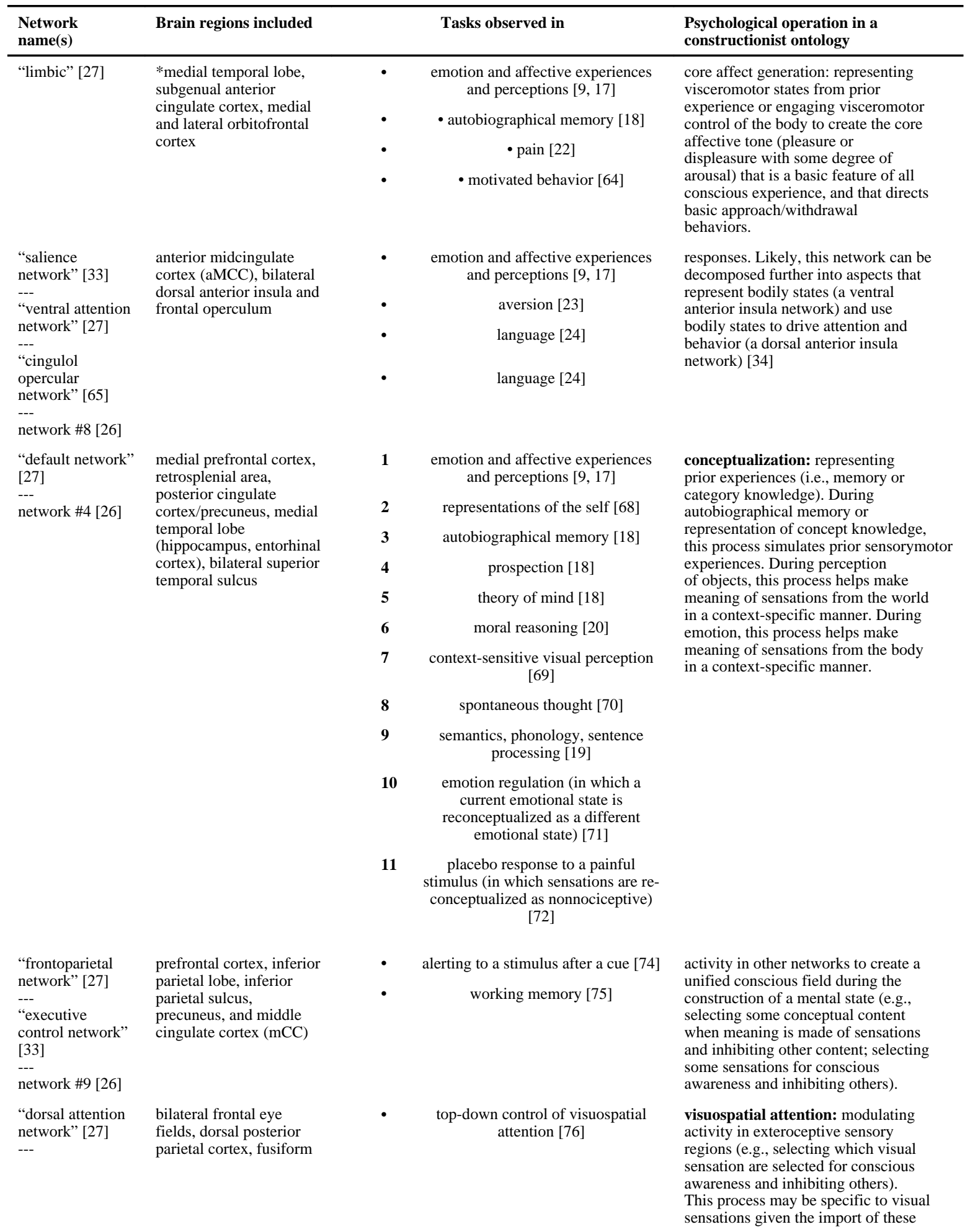

Trends Cogn Sci. Author manuscript; available in PMC 2013 November 01. 


\begin{tabular}{|c|c|c|c|c|}
\hline $\begin{array}{l}\text { Network } \\
\text { name(s) }\end{array}$ & Brain regions included & & Tasks observed in & $\begin{array}{l}\text { Psychological operation in a } \\
\text { constructionist ontology }\end{array}$ \\
\hline network \#9 [26] & gyrus, area MT+ & & & sensations in human evolution. \\
\hline $\begin{array}{l}\text { "sensorimotor" } \\
{[27]}\end{array}$ & $\begin{array}{l}\text { precentral and } \\
\text { postcentral gyri } \\
\text { (sensorimotor cortex), } \\
\text { Heschl's gyrus (primary } \\
\text { auditory cortex) cortex, } \\
\text { posterior insula }\end{array}$ & • & $\begin{array}{c}\text { audition [77] } \\
\text { somatovisceral sensation [78] }\end{array}$ & $\begin{array}{l}\text { exteroceptive sensory perception: } \\
\text { representing auditory and tactile } \\
\text { sensations }\end{array}$ \\
\hline "visual” [27] & occipital lobe & - & vision [79] & $\begin{array}{l}\text { exteroceptive sensory perception: } \\
\text { representing visual sensations }\end{array}$ \\
\hline
\end{tabular}

Note: By examining how brain networks perform during emotions as well as mental states that are typically considered to be other psychological faculties (e.g., memory, perception, theory of mind, etc.), it is possible to use a more robust form of reverse inference to hypothesize the "lowest common denominator" that is the best psychological description for each network. The result is a hypothesis about the functional architecture of the brain that is rooted in basic psychological processes (rather than the set of commonsense faculties now in use; On the need for a new functional architecture in cognitive neuroscience, see $[46,49,52])$. *Although Yeo et al. did not include subcortical structures in their analysis, we include subcortical structures in this network based on their known anatomical connections. We include the nuclei of the basal ganglia, which are involved in orchestrating effortful behavior and motor control [80]. We also hypothesize that the central nucleus of the amygdala, which is involved in producing autonomic responses [80], and the midbrain periacqueductal gray, which is involved in coordinating coherent physiological and behavioral responses [80] are part of this network. The basal ganglia, the amygdala and the periacqueductal gray all project to the ventromedial prefrontal cortex (vmPFC), the major cortical site in Yeo et al.'s limbic network. 